\title{
Malignant ascites drainage with indwelling abdominal catheters: can we predict and prevent infection complication?
}

\author{
Maciej Stukan \\ Department of Gynecologic Oncology, Gdynia Oncology Center, Pomeranian Hospitals, Gdynia, Poland \\ Correspondence to: Maciej Stukan. Department of Gynecologic Oncology, Gdynia Oncology Center, Pomeranian Hospitals, ul. Powstania \\ Styczniowego 1, 81-519 Gdynia, Poland. Email: maciej.stukan@gmail.com. \\ Provenance and Peer Review: This article was commissioned by the Editorial Office, Annals of Palliative Medicine. The article did not undergo external \\ peer review. \\ Comment on: Chan PC, Cheung KWA, Chan CH, et al. Patterns and infection outcomes of bacterial colonization in patients with indwelling \\ abdominal drains for malignant ascites. Ann Palliat Med 2019. [Epub ahead of print].
}

Submitted Nov 16, 2019. Accepted for publication Nov 26, 2019.

doi: 10.21037/apm.2019.11.29

View this article at: http://dx.doi.org/10.21037/apm.2019.11.29

Malignant ascites is an abnormal accumulation of fluid in the peritoneal cavity as a result of cancer. Its occurrence has been reported in numerous malignancies. Ascites is a sign of advanced disease and poor prognosis, with only $11 \%$ of patients surviving $>6$ months, except those with ovarian cancer. Patients with ascites experience numerous significant symptoms that deteriorate their quality of life. First-line interventions beyond treatment of the primary disease include dietary restriction, use of diuretics, and repeated paracentesis. Permanent insertion of catheters in the abdominal cavity is considered to avoid repeated paracenteses and multiple hospitalizations. Fluid drained from the peritoneal cavity can be directed evacuated outside the body, or directed to other body compartments. In the first case, evacuation of the ascitic fluid can be performed using percutaneous catheters inserted directly through the abdominal wall into the peritoneal cavity (e.g., peritoneal and venous catheters), or drains with a subcutaneous tunnel (e.g., PleurX, Tenckhoff, a peritoneal port) (1).

A low risk of insertion-related complications and improvement in symptoms have been reported following the successful insertion of a drain and drainage of ascites (1). Possible long-term adverse events of ascites drainage are infection, fluid leakage, hypotension, renal impairment, electrolyte imbalance, catheter obstruction or dislodgment (1). Some complications can be classified as technical problems that can be easily resolved, while others are caused by the advanced stage of malignancy (rather not ascites-drainagerelated). Infections are the most important complications, and the most difficult to predict and manage. In particular, peritonitis may be fatal. A study conducted by Chan et al. (2) adds valuable evidence on this matter. They performed a retrospective study investigating the patterns of bacterial colonization and the subsequent infection outcomes in patients using indwelling abdominal drains for intermittent drainage of malignant ascites. Patients were managed at the outpatient ascites clinic led by medical professionals. Sixty-nine patients (from a total of 143 patients using abdominal drains; $48.3 \%$ ) who developed bacterial colonization without immediate infection were followed up. The end-point was infection defined as one of the following outcomes: drain-site cellulitis, drain-related peritonitis, fever or sepsis without other demonstratable foci and infected ascites fluid, and physician-diagnosed drain-related infection (i.e., patients without any of the aforementioned clinical features but receiving antibiotics). Correlations between drain-related infection outcomes and patient demographic data, comorbidities (Charlson index), palliative performance status, symptoms burden (Edmonton Symptom Assessment System), information regarding the diagnosis of cancer, drain condition, and details of each drainage were performed. Ascitic fluid specimens for cell count, Gram stain, and bacterial culture were routinely collected on a weekly basis during follow-up.

The study population reflects the reality of a palliative care unit. The median age of patients was 63 years, while their median palliative performance status level was $60 \%$ with significant comorbidities (median Charlson index: 6). 
Different cancers were diagnosed, and the majority of patients $(77 \%)$ had metastatic disease. Notably, one-third of the patients had hepatocellular carcinoma (HCC), and $27.5 \%$ of the patients had comorbid liver cirrhosis. Central venous catheters and pigtail drains were inserted into the abdominal cavity of patients for ascites drainage and in-situ (prior to the first documentation of positive ascitic fluid culture) in $76.8 \%$ and $23.2 \%$ of patients, respectively.

Of the 143 patients receiving this service, 69 were eligible for the study. Thirty patients developed drainrelated infection, accounting for $43.5 \%$ of those with prior positive bacterial colonization of the drain and $21 \%$ of all patients.

Patients underwent a median of two (range, 0-35) sessions of drainage, performed on average 1.3 times per week (range, $0-2.8$ times). The average volume of drained fluid in each session was $1,756 \mathrm{~mL}$. In these conditions the median duration from insertion of the drain to the development of bacterial colonization was 18.0 days (range, 5-159 days). Subsequent drain-related infection developed after a median of 14.5 days (range, 3-117 days). In terms of serious infection outcomes, peritonitis was diagnosed in five of the patients (16.7\%), corresponding to $7.2 \%$ of the entire study population. Four of these patients expired due to drain-related infection, accounting for $80 \%$ of deaths after the diagnosis of peritonitis, and $13.3 \%$ of deaths among all patients who developed drain-related infection. Other infection outcomes were categorized as drainsite cellulitis (20\%), infected ascitic fluid without clinical features of peritonitis (26.7\%), fever or sepsis without other demonstrable foci $(10 \%)$, and physician-diagnosed drainrelated infection $(36.7 \%)$. Of note, there was no mortality related to those outcomes.

In a multivariate analysis, the diagnosis of HCC [odds ratio (OR): 8.85 ; 95\% confidence interval (CI): 1.86-42.07, $\mathrm{P}=0.006]$ and decrease in body weight (OR: 1.20; 95\% CI: $1.02-1.42, \mathrm{P}=0.03)$ were the only two significant factors that positively correlated with infection outcomes. In a univariate analysis, undergoing $\geq 3$ sessions of ascites drainage prior to documentation of bacterial colonization $(\mathrm{P}=0.03)$ was significantly correlated with the development of drain-related infection. The results of the surveillance ascitic fluid culture showed that the presence of Escherichia coli $(\mathrm{P}=0.04)$ and Bacillus species $(\mathrm{P}=0.04)$ in the culture was significantly correlated with infection outcomes (univariate analysis).

Authors (2) ought to be appreciated for presenting very unique data regarding the patterns of infection outcomes of bacterial colonization of catheters inserted into the abdominal cavity of patients for the management of malignant ascites. The methodology and aims of the study were satisfactorily designed and the number of included cases was relatively large; thus, the results are clear and relevant/important. The most useful practical information includes knowledge regarding the proportion of patients in whom a catheter-related infection may occur; the time from colonization to the development of infection; the type and severity of infection outcomes; and the number and type of bacteria culture results.

There are few extremely important and clinically useful issues that should be underlined. Firstly, factors such as age, performance status, comorbidities, intensification of symptoms prior to ascites drainage, and presence of a malignancy other than HCC, were not predictive of infection outcomes. Secondly, almost half of the patients had $\geq 3$ positive ascitic fluid cultures prior to the infection end-point. This indicates that, in the absence of clinical signs of infection, immediate removal of the drain may not be necessary. However, one should reconsider the pros and cons of the drainage individually for each patient depending on her/his condition, and inform the patient and caregivers regarding the potential risks associated with prolonged drainage in such cases. Thirdly, almost all of these ascitic fluid specimens obtained during the infection episodes yielded types of bacteria that were present in at least one of the previous surveillance ascites fluid culture. This demonstrates that, in the case of clinical manifestation of infection, we should be able to initiate aimed treatment with antibiotics.

Patients with malignant ascites have generally poor prognosis, and most exhibit a poor performance status. Clinical decisions are based on a balance between selecting the most appropriate management option to improve patient outcome and estimating the risks associated with the corresponding procedures. Achieving this balance is especially important in this population of patients. Based on the available evidence, it could be concluded that the most important factors for predicting infection complications are liver involvement (HCC or massive liver involvement, or concomitant cirrhosis) and decrease in body weight (prior to ascites drainage).

The study has some limitations that need to be addressed. The frequency of infection was high: $21 \%$ of the entire population and $48 \%$ of patients with a positive ascitic fluid bacterial culture. This finding is in discordance with the data previously reported among patients with 
malignant ascites and undergoing ascites drainage (1): local infection (G2) $0-1.9 \%$ and peritonitis/sepsis (G3) $0-5.7 \%$ (different drains and catheters). It is also inconsistent with the evidence reported following the use of a central venous catheter for malignant ascites drainage: $0-2.3 \%$ peridrain infection and absence of peritonitis/sepsis complications $(3,4)$. It is even more important to notice the high frequency of infection reported in the study conducted by Chan et al. (2), because all patients underwent ascites drainage in a specialized clinic, under the supervision of a medical professional. Other study reported a significantly lower frequency of infections in patients performing drainage unassisted at home (4). Performing drainage procedures in a medical facility may contrary exert an unfavorable effect on the final outcome, because hospitalization is a risk factor for the development of infection. Furthermore, details regarding the type of catheters used may be of significance.

The high frequency of infection observed by Chan et al. (2) may be partly attributable to the large proportion of patients with HCC included in the study. The investigators noticed that patients with HCC having ascites resemble those with ascites secondary to liver cirrhosis. They adequately discussed this observation, recognizing the different pathogenesis of "liver-related" and "peritoneumrelated" ascites formation. Piano et al may have provided a reasonable explanation (5). Ascites represents the most common decompensating event in patients with liver cirrhosis. The appearance of ascites is strongly related to portal hypertension, which leads to splanchnic arterial vasodilation, reduction in the effective circulating volume, activation of endogenous vasoconstrictor systems, and marked retention of sodium and water in the kidneys. Bacterial translocation further worsens hemodynamic alterations in patients with cirrhosis and ascites. Ascites in patients with liver cirrhosis is associated with a high risk of developing further complications of cirrhosis (e.g., spontaneous bacterial peritonitis) (5) that may often be fatal (6). It has been reported that acid suppressive and betaadrenergic antagonist therapies are strongly associated with spontaneous bacterial peritonitis in at-risk individuals with liver cirrhosis. Currently, the use of specific antibiotics for peritonitis should be considered in individuals with cirrhosis and ascites (6). Thus, the administration of concomitant medication is important for the final outcome. There are more data on this matter-the risk of peritonitis during ascites drainage was higher in patients with end-stage liver disease $(8.3 \%)$ (7) than in those with malignancies $(2.5 \%)(8)$. In a series of 24 patients with malignant ascites and 30 with ascites secondary to liver cirrhosis, managed using peritoneo-vascular shunts, septic complications were only observed in the cirrhotic patient group (9). Based on the research discussed herein, we should acknowledge a difference in the risk of infection during ascites drainage, between patients with ascites secondary to massive liver involvement (e.g., HCC, liver metastases) versus peritoneal carcinomatosis. These patients should be independently analyzed in future research studies.

Chan et al. did not evaluate infection outcomes in patients with catheters inserted for malignant ascites drainage, who had no catheter bacterial colonization. Thus, we are unable to determine the total frequency and type of infection outcomes in the entire population. The assumption that patients without bacterial colonization do not develop drain-related infection would be false. Thus, in future research, all patients undergoing drainage of malignant ascites should be followed up for infection outcomes.

Moreover, none of the patients received chemotherapy during drainage of ascites; hence, it is not possible to assess the risk of infection in this important population. The notion that chemotherapy may elevate the risk of drainrelated infection is based merely on assumption. A study involving a small population of patients did not reveal an association between chemotherapy and the risk of drainrelated infection (4). Thus, future research should also focus on patients receiving chemotherapy.

There is strong evidence regarding the use of prophylactic procedures and tools to prevent catheterrelated bloodstream infection, following the insertion of central venous catheters into large vessels (they are designed for this purpose). For patients with catheter inserted into vessels and undergoing hemodialysis, different antibiotic, antimicrobial, and combined (antibioticnon antibiotic) lock solutions decreased the incidence of catheter-related infection versus control lock solutions (10-12). Moreover, available evidence suggests that the insertion of central venous catheters impregnated with antimicrobial agents in large vessels may reduce the rates of central line-associated bloodstream infection, especially in high-risk subgroups $(13,14)$. The most effective devices in preventing infection were the catheters impregnated with minocycline-rifampicin (13). Notably, this approach has alarmed some investigators regarding the development of resistance to the antimicrobial agents $(12,13)$. This is less relevant for patients with malignant ascites, given that their general prognosis is significantly worse than that of 
patients undergoing hemodialysis. Also, catheter dressings and methods of catheter fixation are important. In a meta-analysis involving 6,028 patients, catheter dressings impregnated with chlorhexidine reduced significantly the risk of catheter colonization (OR: 0.46; 95\% CI: 0.36-0.58), decreasing the incidence of catheter-related bloodstream infection (OR: 0.60; 95\% CI: 0.42-0.85) (15). A Bayesian network meta-analysis involving 8,494 patients and evaluating 13 dressings showed that use of a transparent dressing may be the most appropriate method for preventing the development of catheter-related bloodstream infection. Suture and bordered polyurethane dressings may be linked to the lowest risk of infection per 1,000 catheterdays, while sutureless securement devices may lead to the lowest incidence of catheter failure (16). A Cochrane review included five randomized clinical trials (2,277 participants) comparing the frequencies of change of central venous catheters dressings. The analysis indicated that the available evidence was inconclusive regarding the effects of longer or shorter intervals between changes of catheter dressings on the rate of catheter-related infection, mortality, or pain (17).

In conclusion, the study conducted by Chan et al. is an important step forward in understanding the risk of bacterial colonization of catheters and subsequent clinical infection outcomes in patients undergoing repeated drainage of malignant ascites. These findings provide clinicians with important information regarding the possible time and predictive factors associated with the development of drainage-related infection. A prospective trial addressing all aforementioned topics is warranted.

\section{Acknowledgments}

Funding: None.

\section{Footnote}

Conflicts of Interest: The author has no conflicts of interest to declare.

Ethical Statement: The author is accountable for all aspects of the work in ensuring that questions related to the accuracy or integrity of any part of the work are appropriately investigated and resolved.

Open Access Statement: This is an Open Access article distributed in accordance with the Creative Commons Attribution-NonCommercial-NoDerivs 4.0 International
License (CC BY-NC-ND 4.0), which permits the noncommercial replication and distribution of the article with the strict proviso that no changes or edits are made and the original work is properly cited (including links to both the formal publication through the relevant DOI and the license). See: https://creativecommons.org/licenses/by-nc-nd/4.0/.

\section{References}

1. Stukan M. Drainage of malignant ascites: patient selection and perspectives. Cancer Manag Res 2017;9:115-30.

2. Chan PC, Cheung KWA, Chan CH, et al. Patterns and infection outcomes of bacterial colonization in patients with indwelling abdominal drains for malignant ascites. Ann Palliat Med 2019. [Epub ahead of print].

3. Mercadante S, Intravaia G, Ferrera P, et al. Peritoneal catheter for continuous drainage of ascites in advanced cancer patients. Support Care Cancer 2008;16:975-8.

4. Stukan M, Lesniewski-Kmak K, Wroblewska M, et al. Management of symptomatic ascites and post-operative lymphocysts with an easy-to-use, patient-controlled, vascular catheter. Gynecol Oncol 2015;136:466-71.

5. Piano S, Tonon M, Angeli P. Management of ascites and hepatorenal syndrome. Hepatol Int 2018;12:122-34.

6. Dever JB, Sheikh MY. Review article: spontaneous bacterial peritonitis--bacteriology, diagnosis, treatment, risk factors and prevention. Aliment Pharmacol Ther 2015;41:1116-31.

7. Solbach P, Honer Zu Siederdissen C, Taubert R, et al. Home-based drainage of refractory ascites by a permanent-tunneled peritoneal catheter can safely replace large-volume paracentesis. Eur J Gastroenterol Hepatol 2017;29:539-46.

8. Wong BC, Cake L, Kachuik L, et al. Indwelling Peritoneal Catheters for Managing Malignancy-Associated Ascites. J Palliat Care 2015;31:243-9.

9. Söderlund C. Denver peritoneovenous shunting for malignant or cirrhotic ascites. A prospective consecutive series. Scand J Gastroenterol 1986;21:1161-72.

10. Arechabala MC, Catoni MI, Claro JC, et al. Antimicrobial lock solutions for preventing catheter-related infections in haemodialysis. Cochrane Database Syst Rev 2018;4:CD010597.

11. Zhao T, Liu H, Han J. Ethanol lock is effective on reducing the incidence of tunneled catheter-related bloodstream infections in hemodialysis patients: a systematic review and meta-analysis. Int Urol Nephrol 2018;50:1643-52. 
12. Zhang J, Wang B, Li R, et al. Does antimicrobial lock solution reduce catheter-related infections in hemodialysis patients with central venous catheters? A Bayesian network meta-analysis. Int Urol Nephrol 2017;49:701-16.

13. Chong HY, Lai NM, Apisarnthanarak A, et al.

Comparative Efficacy of Antimicrobial Central Venous Catheters in Reducing Catheter-Related Bloodstream Infections in Adults: Abridged Cochrane Systematic Review and Network Meta-Analysis. Clin Infect Dis 2017;64:S131-40.

14. Kramer RD, Rogers MA, Conte M, et al. Are antimicrobial peripherally inserted central catheters associated with reduction in central line-associated bloodstream infection? A systematic review and meta-analysis. Am J Infect
Control 2017;45:108-14.

15. Wei L, Li Y, Li X, et al. Chlorhexidine-impregnated dressing for the prophylaxis of central venous catheterrelated complications: a systematic review and metaanalysis. BMC Infect Dis 2019;19:429.

16. Dang FP, Li HJ, Tian JH. Comparative efficacy of 13 antimicrobial dressings and different securement devices in reducing catheter-related bloodstream infections: A Bayesian network meta-analysis. Medicine (Baltimore) 2019;98:e14940.

17. Gavin NC, Webster J, Chan RJ, et al. Frequency of dressing changes for central venous access devices on catheter-related infections. Cochrane Database Syst Rev 2016;2:CD009213.
Cite this article as: Stukan M. Malignant ascites drainage with indwelling abdominal catheters: can we predict and prevent infection complication? Ann Palliat Med 2020;9(2):136-140. doi: 10.21037/apm.2019.11.29 